\title{
Effects of sublethal exposure to metofluthrin on the fitness of Aedes aegypti in a domestic setting in Cairns, Queensland
}

\author{
Tamara S. Buhagiar $^{1 *}$, Gregor J. Devine ${ }^{2}$ and Scott A. Ritchie ${ }^{1}$
}

\begin{abstract}
Background: Metofluthrin is highly effective at reducing biting activity in Aedes aegypti. Its efficacy lies in the rapid onset of confusion, knockdown, and subsequent kill of a mosquito. In the field, there are a variety of scenarios that might result in sublethal exposure to metofluthrin, including mosquitoes that are active at the margins of the chemical's lethal range, brief exposure as mosquitoes fly in and out of treated spaces or decreasing efficacy of the emanators with time. Sublethal effects are key elements of insecticide exposure and selection.
\end{abstract}

Methods: The metofluthrin dose for each treatment group of male and female Ae. aegypti was controlled using exposure time intervals to a 10\% active ingredient (Al) metofluthrin emanator. Room size and distance from the emanator for all groups was maintained at $3 \mathrm{~m}$. In bioassay cages, male Ae. aegypti were exposed at 0, 5, 10, 20, 30 and 40-min intervals. Females were exposed in bioassay cages at 0, 10, 20, 30, 40 and 60-min intervals. Mortality rates and fecundity were observed between the exposure time groups for both sexes.

Results: Female Ae. aegypti exposed for 60 min had a significantly higher mortality rate (50\%), after a 24-h recovery period, than other exposure times, 10, 20, 30 and $40 \mathrm{~min}(P<0.001)$. An overall difference in fecundity was not observed in females between treatments. A significant effect on male mortality was only observed at 40 min exposure times, three meters from the $10 \% \mathrm{Al}$ emanator $(X=98 \%, P<0.001)$. Males that survived metofluthrin exposure were as likely to produce viable eggs with an unexposed female as males that had not been exposed $(P>0.05)$.

Conclusion: Regardless of sex, if a mosquito survived exposure, it would be as biologically successful as its unexposed counterpart. Portability of the metofluthrin emanator and delayed knockdown effects create opportunities for sublethal exposure and potential pyrethroid resistance development in Ae. aegypti, and should be taken into consideration in recommendations for field application of this product, including minimum exposure periods and a prescribed number of emanators per room based on volume.

Keywords: Metofluthrin, Spatial repellents, Pyrethroid-resistance, Aedes aegypti, Dengue

\section{Background}

The primary vector of dengue in north Queensland, and in most parts of the tropical regions of the world, is the yellow fever mosquito, Aedes aegypti (L.) [1, 2]. This day-biting mosquito has a close association with the domestic environment and is often described as an "urban mosquito" [3-5].

\footnotetext{
* Correspondence: Tamara.Buhagiar@jcu.edu.au

${ }^{1}$ College of Public Health, Medical and Veterinary Sciences, James Cook

University, PO Box 6811QLD, Cairns 4870, Australia

Full list of author information is available at the end of the article
}

Mosquito coils and vaporizer mats are well-documented spatial repellents that have been used in repelling mosquitoes from human biting $[6,7]$. Unfortunately, both of these products are limited by their requirement for a heat source to vaporize their active ingredient, efficacy $[7,8]$, and their limited use indoors [9]. Studies have shown varying susceptibility to pyrethroid-based mosquito coils between different mosquito species, and within a species, specifically Ae. aegypti [7, 8]. Increased efficacy of pyrethroid-based mosquito coils against Ae. aegypti is observed with the addition of a synergist [8]. Use of synergists, such as Octachlorodipropyl ether (S-2) are commonly 
used in Asia, but are illegal in the USA [9], as it exposes humans to some levels of bio-chloromethyl ether (BCME), an extremely potent lung carcinogen. A study by Liu et al., [10] found that burning one mosquito coil produced the same amount of fine and ultrafine particulate matter mass as burning 75-137 cigarettes. They also found a large suite of volatile organic compounds, including carcinogens and suspected carcinogens in the coil smoke [10].

Investigations into the insecticidal activity of norchrysanthemic acid esters with high vapor activity at ambient temperature [11] have resulted in the identification of an effective synthetic pyrethroid commonly known as metofluthrin. This compound does not require a heat source or burning of carcinogenic material, making it a suitable candidate for use indoors.

Metofluthrin is a volatile pyrethroid and has been shown to be extremely effective at reducing biting activity in Aedes species, including Ae. aegypti, as well as Culex quinquefasciatus in the field [12-14]. Metofluthrin has been shown not to have any repellent or expellant effects with the $5-10 \%$ active ingredient (AI) passive formulations used by Rapley et al. [6], and Ritchie \& Devine [15]. Biting reduction with metofluthrin is primarily achieved through the compound's ability to confuse, knockdown, and kill Ae. aegypti $[6,15]$. Due to its unique and effective mechanism for reducing biting, it could serve as an effective tool to disrupt biting by $A e$. aegypti and potentially reduce dengue transmission [15].

Metofluthrin is a volatile pyrethroid that has insecticidal properties and well-documented behavioral effects on resting and biting $[6,15]$. However, due to its insecticidal properties, portability, limits in spatial range, and delayed onset of knockdown and lethal effects, an opportunity is created for sublethal exposure of mosquitoes. In this study, we look at the impacts of sublethal exposure in semi-field conditions, controlled through exposure time, on adult Ae. aegypti fitness. To date, the physiological effects of sublethal exposure on Ae. aegypti, if any, have not yet been described. A study involving the exposure of Ae. aegypti to sublethal doses of deltamethrin, an insecticidal pyrethroid, found that unmated females were more likely to escape and less likely to blood feed than mated females in the presence of a host [16]. Cohnstaedt \& Allan [17] found a significant reduction in the response of $A e$. aegypti, Culex quinquefasciatus, and Anopheles albimanus to host attractants when exposed to a sublethal dose $\left(\mathrm{LD}_{25}\right)$ of permethrin and deltamethrin for a $24 \mathrm{~h}$ period. Exposure of Aedes vigilax larvae to sublethal doses $\left(\mathrm{LD}_{50}\right)$ of methoprene significantly reduced survival in adult females and males, and reduced blood-feeding success [18]. In a study by Wagman et al. [19], prolonged sublethal exposure of wild $\mathrm{F}_{0} A$ e. aegypti that were not responsive to the repellent effects of transfluthrin demonstrated decreased susceptibility to transfluthrin toxicity by $\mathrm{F}_{4}$.
Methoprene's insecticidal mechanism is inherently different from transfluthrin, permethrin, and deltamethrin, however, the principal remains the same. There are a variety of scenarios that might result in sublethal exposure to metofluthrin, including mosquitoes that are active at the margins of the chemical's lethal range, periodic exposure as mosquitoes fly in and out of treated spaces or decreasing efficacy of the emanators with time. Sublethal effects are key elements of insecticide exposure and selection. In this study, we evaluate the impact of sublethal exposure to metofluthrin on the bloodfeeding, mating success, and egg quality of adult Ae. aegypti. Determining the impacts of sublethal exposure on overall mosquito fitness and fecundity will highlight the potential for heritable insensitivity, as described by Wagman et al. [19] and pyrethroid resistance in wild, pyrethroid susceptible Ae. aegypti.

\section{Methods}

The metofluthrin product used in this study was a small $(9.5 \times 15 \times 1 \mathrm{~cm})$ plastic frame containing a polyethylene mesh in which the 10\% AI formulation is incorporated into (Sumitomo Chemical Australia Pty Ltd., Sydney, Australia) [15] (Fig. 1a). In previous longevity studies of this emanator, its efficacy was sustained up to 20 days [15].

\section{Rearing, sexing, caging, and blood-feeding mosquitoes}

A Cairns colony (F1) of Ae. aegypti infected with wMel Wolbachia, derived from field populations, was used throughout the experiments. The colony was reared in a controlled temperature room at $25{ }^{\circ} \mathrm{C}$ and maintained at $70 \%$ relative humidity. Wolbachia-infected mosquitoes were used to avoid accidental introduction of uninfected mosquitoes in an area where the Eliminate Dengue program had established $w \mathrm{Mel}$ in Ae. aegypti. In the experiments requiring virgin females, mosquitoes were separated as pupae by size and placed in cohorts of ten. Once they had emerged, and it was certain that the females were not exposed to a male, they were then placed into a BugDorm $(30 \times 30 \times 30 \mathrm{~cm})$ with other virgin females, and provided with a $10 \%$ honey solution. When mosquitoes were required to take a blood meal, honey pads were removed $24 \mathrm{~h}$ prior. When blood meals were required, mosquitoes were offered a blood meal by resting the back of a human leg on top of the cage or container (Human Ethics Approval from James Cook University, H6286).

\section{Experimental setting}

All experiments took place within a large living room $\left(111 \mathrm{~m}^{3}\right)$ within a Queenslander-style house in Cairns, Queensland, Australia (Fig. 1b). The 10\% AI metofluthrin 


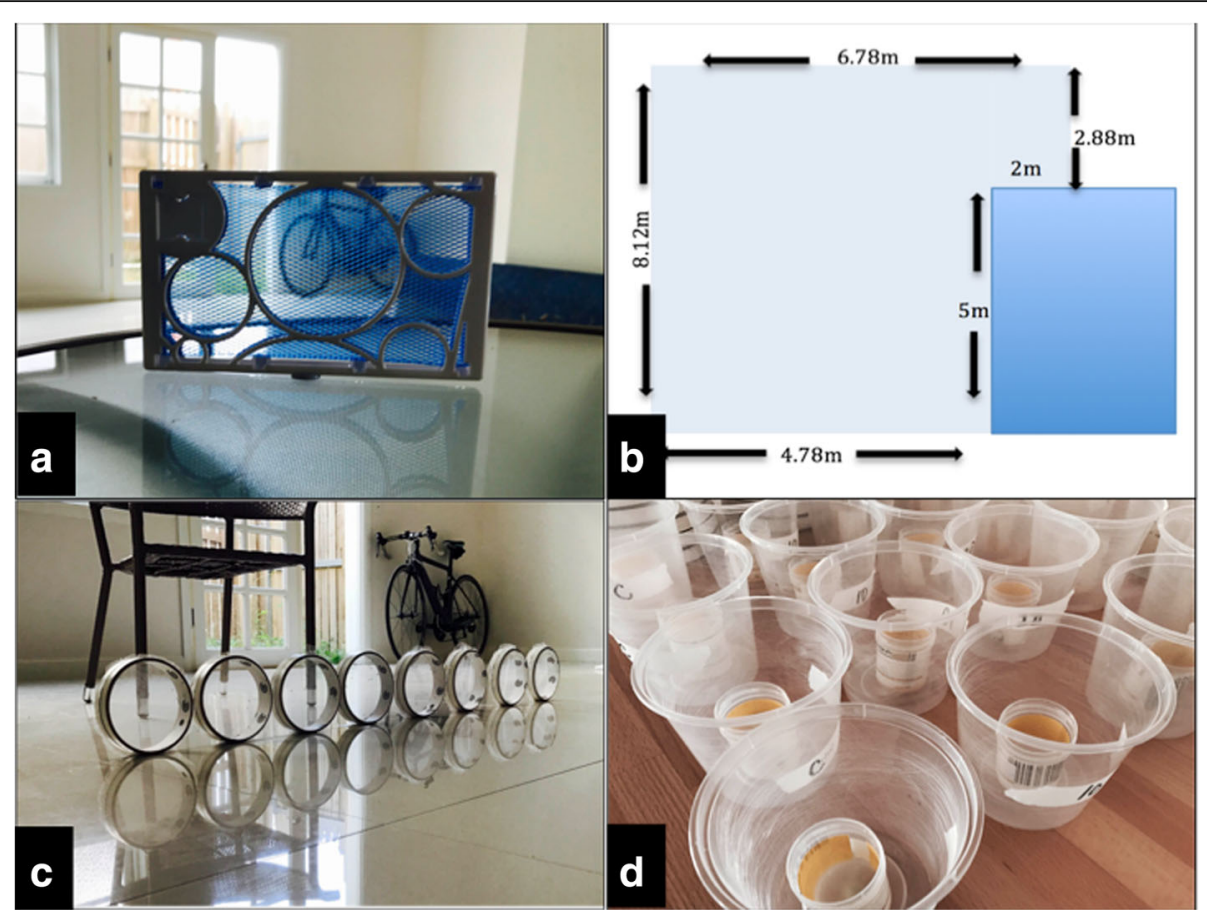

Fig. 1 a 10\% Al metofluthrin emanator (Sumitomo). b Floor plan of the experimental room. c Female mosquitoes in tambourine cages. d Containers where female cohorts were placed post-exposure

emanator was placed centrally within the room and allowed to volatilize for $30 \mathrm{~min}$ prior to the commencement of each experimental repetition.

\section{Effects on fitness and fecundity: females}

Cohorts of approximately 10 mated female Ae. aegypti were placed into fine mesh tambourine cages (Fig. 1c) and exposed in the metofluthrin-treated room at $3 \mathrm{~m}$ from the emanator for 10, 20, 30, 40 and $60 \mathrm{~min}$. The control group, 0 min, was kept in an unexposed room within the same dwelling. Once exposed, the knockdown rate was recorded for each exposure time. Each female cohort was then placed into $500 \mathrm{ml}$ plastic container. The containers were roughened on the inside to ensure the mosquito could easily rest on the walls. Inside the containers, was a smaller $100 \mathrm{ml}$ cup with a sand paper egg-laying strip, half-submerged in water. The containers were covered with a muslin cloth. The containers were transparent, and mosquitoes could be easily observed without being removed (Fig. 1d).

Mortality was observed after 3 and 24 h post-exposure. A blood meal was offered to each cohort at $24 \mathrm{~h}$ for $5 \mathrm{~min}$, and the proportion of blood-fed mosquitoes was recorded. The egg strips were removed 7 days after blood meal, and stored for $72 \mathrm{~h}$ to embryonate. The eggs were then immersed in water with yeast to induce hatching. After $48 \mathrm{~h}$, the egg strips were examined under a microscope to determine the displacement of the operculum from the egg, an indicator that the egg was viable and had hatched. The mean number of eggs laid per blood-fed female for each treatment and the mean hatch rate was recorded. A total of 6 replicates were completed.

\section{Effects on fitness and mating success: males}

Cohorts of 5 virgin male Ae. aegypti were placed into tambourine cages and exposed to metofluthrin for 5,10 , 20, 30 and $40 \mathrm{~min}$ at $3 \mathrm{~m}$ from the emanator. The control group, 0 min, was kept in an unexposed room within the same dwelling. The knockdown rate was recorded for each treatment. Mortality was observed after $6 \mathrm{~h}$. Surviving individual males were placed individually into a single plastic $250 \mathrm{ml}$ cup containing one virgin female. Each female had previously been bloodfed 3 days prior. Inside each cup was a small $100 \mathrm{ml}$ container with a sand paper egg-laying strip, halfsubmerged in water. The $250 \mathrm{ml}$ cups were then covered with a muslin cloth, and mosquito pairs were given 7 days to mate and produce eggs on the oviposition strip. Eggs laid onto the strip were removed from the water and stored for $72 \mathrm{~h}$ to embryonate, then immersed in water with yeast to induce hatching. After $48 \mathrm{~h}$, egg strips were examined under a microscope to determine the displacement of the operculum from the egg, an indicator that the egg was viable and had hatched. The number of eggs laid and the proportion of eggs hatched were recorded for each male/female pair. 


\section{Data analyses}

\section{Effects on fitness and fecundity: females}

Knockdown and mortality data were arcsine square root transformed and analyzed using a 2-Way ANOVA followed by a Tukey's multiple comparisons test. Blood-feeding and fecundity data were arcsine square root transformed and analyzed using a One-Way ANOVA followed by a Dunnett's multiple comparisons test.

\section{Effects on fitness and mating success: males}

Mortality and mating success data were arcsine square root transformed and analyzed using a One-Way ANOVA. A Holm-Sidak's multiple comparisons test for the mortality data was performed. Hatch rate data were analyzed using a One-Way ANOVA, followed by a Dunnett's multiple comparisons test.

All data were analyzed in Prism 6 for Mac OSX (v. 6.0 h, GraphPad Software Inc.).

\section{Results}

\section{Females}

\section{Knockdown and mortality}

One hundred percent of all female Ae. aegypti exposed to metofluthrin (10\%) for $10 \mathrm{~min}$, or more at $3 \mathrm{~m}$ from the emanator were knocked down (Fig. 2a). Forty-eight per cent of females exposed for $60 \mathrm{~min}$ were dead at $24 \mathrm{~h}$ post-exposure. At 3 and $24 \mathrm{~h}$ post-treatment, a 2-Way
ANOVA found a significant effect of exposure time on mortality, and a significant effect of the amount of time post-exposure on mosquito mortality $\left(F_{(5,30)}=35.67\right.$, $P<0.0001$ and $F_{(1,30)}=15.13, P<0.001$, respectively) (Fig. 2b). A Tukey's post-hoc analysis found that at $3 \mathrm{~h}$ post-treatment, a significant difference in the effect of metofluthrin on mortality was observed between the 60-min exposure treatment and all other treatment groups $(P<0.0001)$. At $24 \mathrm{~h}$ post-exposure, a significant difference in mortality was observed between the control and mosquitoes exposed for $30 \mathrm{~min}$ or more $(P<0.001)$. After $24 \mathrm{~h}$ post-exposure, $50 \%$ of female $A e$. aegypti survived exposure and received a sublethal dose of metofluthrin after $60 \mathrm{~min}$.

\section{Blood-feeding and fecundity}

Female Ae. aegypti that survived metofluthrin exposure between 10 and $60 \mathrm{~min}$ were considered sublethally exposed to metofluthrin (10\% AI) after a $24 \mathrm{~h}$ recovery period. A One-Way ANOVA of transformed blood-feeding data from sublethally exposed females found an overall significant effect of treatment (duration of exposure to metofluthrin) on blood-feeding success $\left(F_{(5,30)}=4.977\right.$, $P=0.002)$. A Dunnett's post-hoc analysis found a significant difference between the control group and mosquitoes sublethally exposed for 30 and $60 \mathrm{~min}$ (Fig. 2c). A OneWay ANOVA of transformed egg hatching data, found no

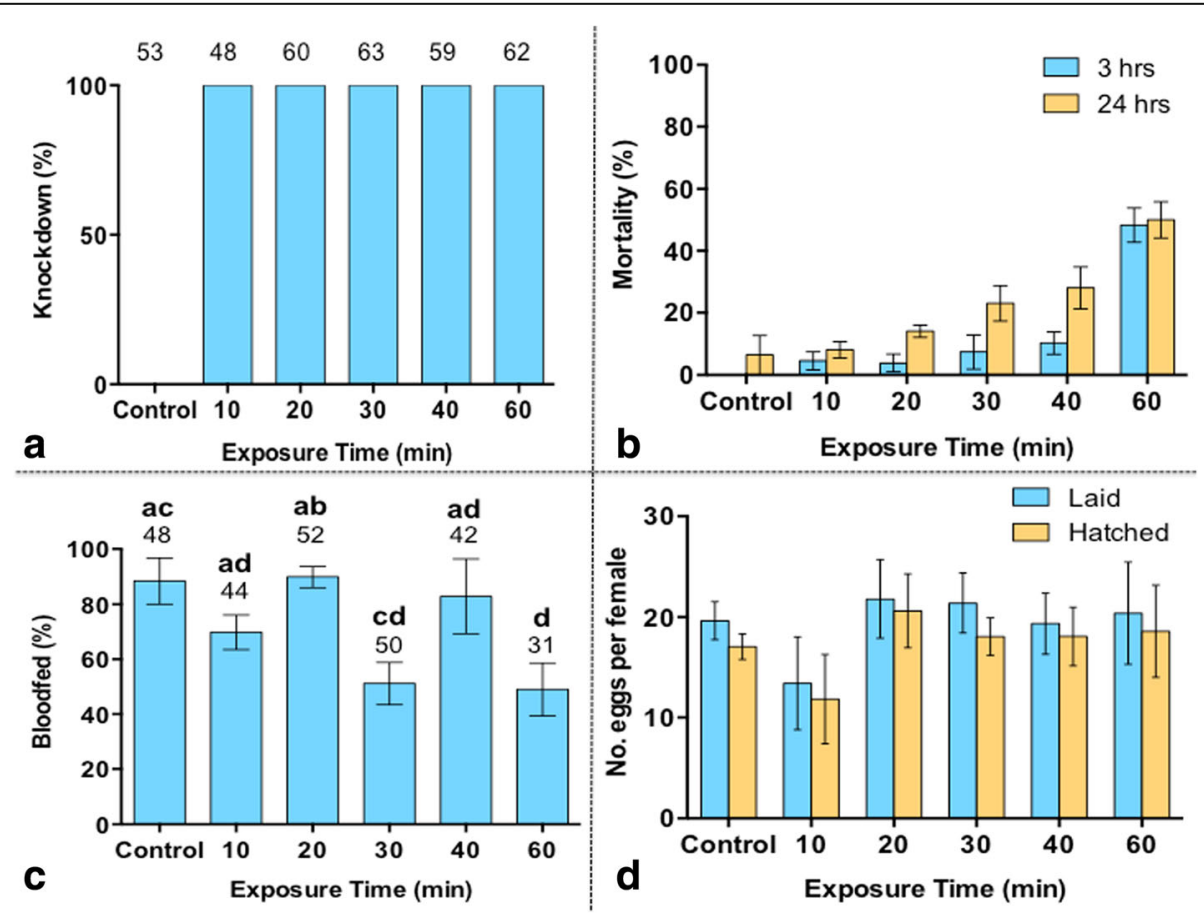

Fig. 2 a Mean percent \pm SE knockdown of female Ae. aegypti exposed to metofluthrin (10\% Al). The number above each column represents the total number of females exposed in each treatment group $(n=6)$. b Mean percent \pm SE mortality of females 3 and 24 h post-exposure to metofluthrin. $\mathbf{c}$ The mean percent \pm SE of blood-fed mosquitoes at $24 \mathrm{~h}$. d Mean percent \pm SE of eggs laid and hatched per treatment. There was no significant effect of treatment on the number of eggs laid per female or the hatch rate of those eggs 
significant effect of treatment on the fecundity of sublethally exposed females $\left(F_{(5,28)}=1.33, P=0.28\right)$. Female Ae. aegypti that survived metofluthrin exposure were as likely to lay viable eggs as each other and as the control (Fig. 2d).

\section{Males}

\section{Knockdown and mortality}

One hundred percent of all male Ae. aegypti exposed to metofluthrin (10\%) for $10 \mathrm{~min}$ or more were knocked down (Fig. 3a). A One-way ANOVA of transformed data found an overall significant effect of treatment on mortality rates amongst treatment groups $\left(F_{(5,18)}=5.415\right.$, $P=0.0033$ ) (Fig. 3b). A Holm-Sidak's multiple comparisons test found no significant difference in mortality between the control $(\bar{X}=0 \%)$ and males exposed up to 30 min (Fig. 3a). However, a significant effect of treatment on mortality was observed between the control $(\bar{X}=0 \%)$ and males exposed for $40 \min (\bar{X}=98 \%)$ $(P<0.001)$ (Fig. 3b).

\section{Mating success}

Males that survived metofluthrin exposure between 5 and $40 \mathrm{~min}$ were considered sublethally exposed. A One-Way ANOVA found no significant effect on mating success between treatment groups $\left(F_{(5,70)}=1.915\right.$, $P=0.103$ ) (Fig. 3c), indicating that regardless of exposure time if a male Ae. aegypti survived metofluthrin exposure, he was as likely to successfully mate as an unexposed male when paired with an unexposed female. A One-Way ANOVA of transformed egg hatching data found an overall significant effect of treatment on egg viability, measured by the hatch rate of the eggs $\left(F_{(4,41)}=3.183\right.$, $P=0.0225)$. A Dunnett's multiple comparisons analysis found a significant difference in egg viability, as measured by the proportion hatched, solely between the control group $(X=0.84)$ and the 20-min exposure treatment $(X=0.47)$ (Fig. 3d).

\section{Discussion}

Metofluthrin causes a reduction in human biting through two main modes of pyrethroid action, firstly as a result of its knockdown effects and secondly due to the disruption in orientation towards the host [14]. Ritchie \& Devine [15] found that exposure to $10 \% \mathrm{AI}$ emanators within small rooms rapidly affected all freeflying Ae. aegypti, reducing human landing counts to "negligible" after eight minutes, and after $20 \mathrm{~min}, 90 \%$ of mosquitoes were dead. Rapley et al. [6] found 98\%
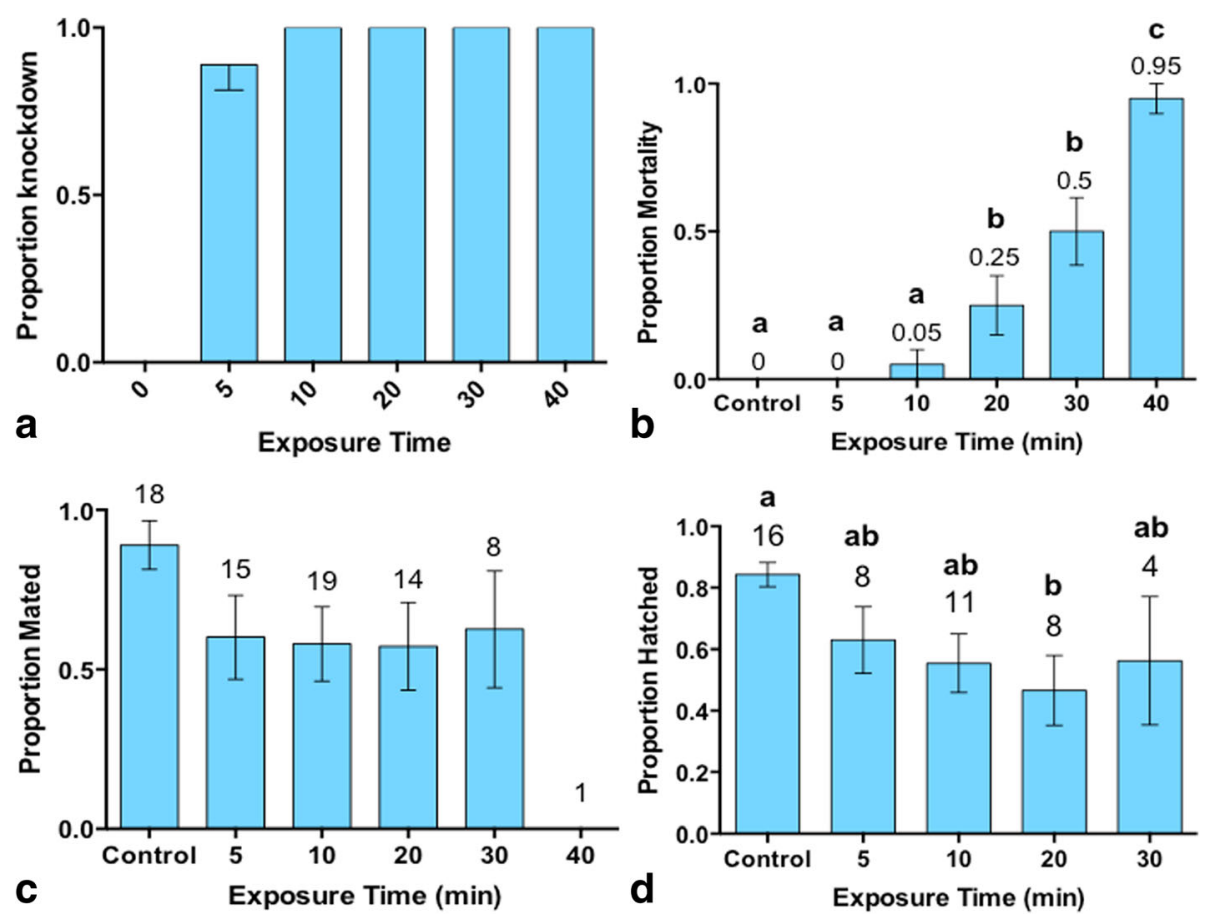

Fig. 3 a Mean \pm SE knockdown rates of male Ae. aegypti exposed to metofluthrin. b Mean \pm SE mortality of male Ae. aegypti exposed to metofluthrin $(10 \% \mathrm{Al})$ for $0,5,10,20,30$ and 40 min exposure periods. Survival significantly decreases after 20 min of exposure. c Mean \pm SE proportion of males that survived metofluthrin exposure and mated successfully. Mating success was confirmed by the production of eggs by the paired female. The number above each column is the $n$-value. No significant difference in mating success was observed between groups. $\mathbf{d}$ Mean proportion \pm SE of eggs that hatched for each successfully mated male/female pair. The number above each column is the $n$-value 
mortality of Ae. aegypti in the presence of metofluthrin (4.1\% AI) after $24 \mathrm{~h}$. In this study, we did not observe the same impacts on mosquito survival at $20 \mathrm{~min}$ as described in Ritchie \& Devine with the same emanator formulation [15]. We believe that this can be accounted for as a result of room size and the exposure of mosquitoes to metofluthrin from within a cage. In this study, the size of the room was $111 \mathrm{~m}^{3}$ versus 22.3 and $24.3 \mathrm{~m}^{3}$ in those used by Ritchie \& Devine [15]. In larger room studies using bioassay cages, effects of metofluthrin $(10 \% \mathrm{AI})$ on mortality in female Ae. aegypti exposed within one meter of the emanator, were not observed until one hour of exposure in rooms that were 41.2 and $37.8 \mathrm{~m}^{3}$ [20]. The mosquitoes used in Ritchie \& Devine [15] were also free flying compared to caged mosquitoes used in Darbro et al. [20] and in the set of experiments undertaken here. The fine mesh of the cages may have provided some protection by restricting airflow into the cage $[15,20]$. Bibbs \& Xue [21] also found limited effective range in a $31.2 \%$ concentration OFF! Clip-on repellent device used outdoors, whereby significant knockdown and mortality effects were not sustained beyond $0.3 \mathrm{~m}$ in caged Ae. aegypti. Although "kill" is a desirable outcome as a result of metofluthrin exposure, it is its immediate impacts on host-seeking activity that is of most importance to the host.

In terms of insecticide resistance, the insecticidal properties of metofluthrin become very important. Evaluating the potential for sublethal exposure of mosquitoes to occur when metofluthrin is used in practice, combined with determining any measurable impacts or lack thereof on Ae. aegypti fitness is essential when determining "best practice" for field application of this product. Insecticides in low or insufficient doses have been shown to have sublethal effects on mosquito fitness, and behavior $[16,18]$, as well as have significant impacts on sensitivity and toxicological vulnerability of the mosquito in subsequent generations [19]. Sublethal conditions were created in these experiments by controlling the exposure time of the mosquitoes and maintaining their distance from the emanator. A mosquito that is not near enough to the emanator or not exposed for a long enough period to be killed can be defined as "sublethally exposed." Excluding sublethal studies from the evaluation of an insecticide could mean that the overall efficacy of a product, when applied, could be greatly underestimated.

Male Ae. aegypti, overall, are more vulnerable than females to metofluthrin exposure, as reflected in their mortality rates, however unlike females, they only need to survive a relatively short period of time, enough to find a female and mate, completing the single act required of that individual to pass its genetic material on to the next generation. Aedes aegypti females, when given the opportunity to recover from knockdown, will, once out of the effective range of an emanator, resume blood-feeding, mating, and ovipositioning with the same success as an unexposed individual. This opportunity to recover could result if, for example, the metofluthrin emanator is removed from the room or a draft blows the knocked down mosquito out of the effective range. Regardless of sex, if a mosquito survived exposure, it was as biologically successful as its unexposed counterpart. Sublethal exposure, as observed in this suite of experiments, appears to have no obvious effect on the fitness of Ae. aegypti, but should be considered in its recommendations for use and deployment.

Metofluthrin-treated devices deliver protected zones. In this experiment specifically, we placed the cages at three meters distance from the emanator, and observed $100 \%$ knockdown within ten minutes of both males and females, rendering them incapable of flying or biting. Its at the margins of the defined effective zones of the metofluthrin emanator, where mosquitoes will be less affected and may fully recover if they are able to orientate away from the chemical source or if the treatment is removed. Since metofluthrin is a confusant, rather than a repellant, this may be a minor issue as it does not expel the mosquito from the treated area, as observed in Ritchie \& Devine [15]. As demonstrated in previous experiments using metofluthrin emanators, mosquitoes entering the effective zone of metofluthrin, are likely to experience a rapid onset of negative effects, significantly affecting their fitness, including flight, host seeking, and biting. These immediate effects on flight and host orientation, in combination with metofluthrin's lack of repellant or expellant properties [15], may be conducive to the mosquito remaining in the effective range, eventually succumbing to its insecticidal effects, particularly indoors. Environments, where sublethal exposure is most likely to occur, are semi-enclosed areas, for example, a covered veranda or a laundry area attached to the home where mosquitoes are able to move out of the effective range more easily.

This study involved controlled exposure of Ae. aegypti to a series of metofluthrin doses determined by exposure time to the metofluthrin emanator, while distance of the mosquitoes from the emanator and the size of the room was maintained. Mortality rates for a series of doses provide a guideline for minimum exposure periods required for sufficient insecticidal impacts on $A$ e. aegypti in the field within an enclosed space. Future investigations involving metofluthrin should be directed toward determining metofluthrin's ability to cause resistance in pyrethroid-susceptible populations of Ae. aegypti, defining its effective range, and development of usage and deployment protocols that maximize its insecticidal impacts, thus reducing opportunities for sublethal exposure. 


\section{Conclusion}

Overall, Ae. aegypti that survive exposure to metofluthrin (sublethal exposure) will be as biologically successful as their unexposed counterparts. Metofluthrin is an insecticide that volatilizes at room temperature, is portable, has a limited spatial range, and delayed knockdown and lethal effects. Portability of the product and delayed knockdown effects create opportunities for sublethal exposure and potential pyrethroid resistance development in Ae. aegypti. The results of our study combined with the described attributes of the metofluthrin emanator should be taken into consideration in recommendations for field application of this product, including minimum exposure periods and a prescribed number of emanators per room based on volume.

\section{Abbreviations}

Al: Active ingredient; SE: Standard error

\section{Acknowledgements}

Thank you to Dr. Luke Rapley for his feedback on the experimental design for components of this project, and to Dr. Tobin Northfield for his advice with the statistics.

\section{Funding}

The study was funded by a Deployed Warfighter Deployment Grant (grant \#W911QY-15-1-0006) held by QIMR Berghofer. Dr. Scott A. Ritchie is supported by the National Health and Medical Research Council. Senior Research Fellowship \#1044698.

\section{Availability of data and materials}

All data generated or analyzed during this study are included in this published article.

\section{Authors' contributions}

Experimental design was created by TB. All experiments were performed by TB. Data was recorded, analyzed, and interpreted by TB. TB is the primary writer of this manuscript, with significant intellectual input from SR and GD. SR and GD contributed significantly to the editing of this manuscript. All authors read and approved the final manuscript.

\section{Competing interests}

The authors declare that they have no competing interests.

\section{Consent for publication}

Not applicable.

\section{Ethics approval and consent to participate}

Human ethics approval was granted by the James Cook University ethics committee, H6286.

\footnotetext{
Author details

${ }^{1}$ College of Public Health, Medical and Veterinary Sciences, James Cook University, PO Box 6811QLD, Cairns 4870, Australia. ${ }^{2}$ Mosquito Control Laboratory, QIMR-Berghofer Institute of Medical Research, QLD, Brisbane 4006, Australia.
}

Received: 19 December 2016 Accepted: 25 May 2017

Published online: 31 May 2017

\section{References}

1. Scott TW, Chow E, Strickman D, Kittayapong P, Wirtz RA, Lorenz LH, et al. Blood-feeding patterns of Aedes aegypti (Diptera, Culicidae) collected in a rural Thai village. J Med Entomol. 1993;30(5):922-7.

2. Scott TW, Clark GG, Lorenz LH, Amerasinghe PH, Reiter P, Edman JD. Detection of multiple blood feeding in Aedes aegypti (Diptera, Culicidae) during a single gonotrophic cycle using a histologic technique. J Med Entomol. 1993;30(1):94-9.

3. Perich MJ, Kardec A, Braga IA, PI F, Burge R, Zeichner BC, et al. Field evaluation of a lethal ovitrap against dengue vectors in Brazil. Med Vet Entomol. 2003;17:205-10.

4. Sithiprasasna R, Mahapibul P, Noigamol C, Perich MJ, Zeichner BC, Burge B, et al. Field evaluation of a lethal ovitrap for the control of Aedes aegypti (Diptera: Culicidae) in Thailand. J Med Entomol. 2003;40(4):455-62.

5. Ritchie SA. Dengue vector bionomics: Why Aedes aegypti is such a good vector. In: Gubler DJ, Ooi EE, Vasudevan S, Farrar J, editors. Dengue and dengue hemorrhagic fever. 2nd edn. Wallingford: CABl; 2014.

6. Rapley LP, Russell RC, Montgomery BL, Ritchie SA. The effects of sustained release metofluthrin on the biting, movement, and mortality of Aedes aegypti in a domestic setting. Am J Trop Med Hyg. 2009;81(1):94-9.

7. Katsuda $Y$, Leemingsawat S, Thongrungkiat S, Prummonkol S, Samung Y, Kanzaki T, et al. Control of mosquito vectors of tropical infectious diseases: (3) Susceptibility of Aedes aegypti to pyrethroid and mosquito coils. SE Asian J Trop Med. 2009;40(5):929-36.

8. Katsuda Y, Leemingsawat S, Thongrungkiat S, Komalamisara N, Kanzaki T, Watanabe T, et al. Control of mosquito vectors of tropical infectious diseases: (1) Bioefficacy of mosquito coils containing several pyrethroids and a synergist. SE Asian J Trop Med. 2008;39(1):48-54.

9. Krieger RI, Dinoff TM, Zhang XF. Octachlorodipropyl ether (S-2) mosquito coils are inadequately studied for residential use in Asia and illegal in the United States. Environ Health Perspect. 2003;111(12):1439-42.

10. Liu W, Zhang J, Hashim JH, Jalaludin J, Hashim Z, Goldstein BD. Mosquito coil emissions and health implications. Environ Health Perspect. 2003; 111(12):1454-60.

11. Ujihara K, Mori T, Iwasaki T, Sugano M, Shono Y, Matsuo N. Metofluthrin: a potent new synthetic pyrethroid with high vapor activity against mosquitoes. Biosci Biotechnol Biochem. 2004;68(1):170-4.

12. Kawada H, Yen NT, Hoa NT, Sang TM, Dan NV, Takagi M. Field evaluation of spatial repellency of metofluthrin impregnated plastic strips against mosquitoes in Hai Phong city. Vietnam Am J Trop Med Hyg. 2005;73(2):350-3.

13. Lucas J, Shono Y, Iwasaki T, Ishiwatari T, Spero N, Benzon G. U.S. laboratory and field trials of metofluthrin (SumiOne) emanators for reducing mosquito biting outdoors. J Am Mosq Contr Assoc. 2007;23(1):47-54

14. Kawada H, Iwasaki T, Loan LL, Tien TK, Mai NTN, Shono Y, et al. Field evaluation of spatial repellency of metofluthrin-impregnated latticework plastic strips against Aedes aegypti (L.) and analysis of environmental factors affecting its efficacy in My Tho City, Tien Giang, Vietnam. Am J Trop Med Hyg. 2006;75(6):1153-7.

15. Ritchie SA, Devine GJ. Confusion, knock-down and kill of Aedes aegypti using metofluthrin in domestic settings: a powerful tool to prevent dengue transmission? Parasit Vectors. 2013;6(1):262.

16. Boonyuan W, Kongmee M, Bangs MJ, Prabaripai A, Chareonviriyaphap T. Host feeding responses of Aedes aegypti (L.) exposed to deltamethrin. J Vector Ecol. 2011;36(2):361-72.

17. Cohnstaedt LW, Allan SA. Effects of sublethal pyrethroid exposure on the hostseeking behavior of female mosquitoes. J Vector Ecol. 2011;36(2):395-403.

18. Ritchie SA, Asnicar M, Kay BH. Acute and sublethal effects of (s)-methoprene on some Australian mosquitoes. J Am Mosq Contr Assoc. 1997:13(2):153-5.

19. Wagman JM, Achee NL, Grieco JP. Insensitivity to the spatial repellent action of transfluthrin in Aedes aegypti: a heritable trait associated with decreased insecticide susceptibility. PLoS Neg Trop Dis. 2015;9(4):e0003726.

20. Darbro JM, Muzari MO, Giblin A, Adamczyk RM, Ritchie SA, Devine GJ. Reducing biting rates of Aedes aegypti with metofluthrin: investigations in time and space. Parasit Vectors. 2017;10(1):69.

21. Bibbs CS, Xue R-D. OFF! Clip-on Repellent Device With Metofluthrin Tested on Aedes aegypti (Diptera: Culicidae) for Mortality at Different Time Intervals and Distances. J Med Entomol. 2016;53(2):480-3. 